\title{
Role of Mobile Handhelds in Redefining How We Work, Live and Experience the World Around Us: Some Challenges and Opportunities
}

\author{
Sanjoy Paul \\ Infosys Technologies Limited \\ India \\ Sanjoy_Paul@infosys.com
}

\begin{abstract}
Mobile handheld devices have been changing form and functionality over the last decade. It has changed from a limited-function device, with the primary function being making phone calls, to a multi-function device that morphs into a media device for watching movies, or into a remote control for home appliances, or into a wallet for making payments. In my presentation, I will argue that the range and diversity of mobile devices will continue to increase, and in addition, mobile handhelds will become extensions of other devices, such as, TV and PC, thereby enabling users to have an immersive experience while consuming services. As a result, techniques that enable cross-channel/device continuity of services will be essential to provide the right user experience leading to the importance of models, techniques and middleware infrastructures that abstract this complexity from application development and delivery perspectives. Developing intelligent mobile applications, such as, augmented reality, that optimize use of context information, such as, location, time, network connectivity, device capability, user behavior, etc. will also become important. I'll also argue that smart experience and not processing power will be the key to success for any mobile device/application. This will require new perspective on how to approach designing mobile solutions. Another viewpoint that will be covered in the presentation is that of using mobile devices as the "gateway" between the physical world and the inter-networked world. This is starting to happen with the explosion of sensors embedded in the physical world, advances in ultra low power short range RF technology and the ubiquity of mobile devices bridging the physical world with the Internet. In that context, I'll discuss how mobile devices, in conjunction with sensors, are now providing context based services to the user, managing their health, providing advisory services, and enabling the "Internet of Things". Finally, I'll talk about three categories of applications: (1) consumer, (2) enterprise and (3) industry-specific. Differences between the requirements of each category of mobile applications will be discussed and examples will be given with real working demos. Challenges in scaling and deploying mobile applications in real-world environment will be highlighted. In addition, privacy and security issues with integrating both personal (consumer) and enterprise applications on the same mobile handheld will be discussed as real business challenges. In that context, the value of end-to-end testing of mobile applications will be highlighted and potential approaches to testing will be discussed. Finally, the talk will end with a discussion around the potential of using a network of mobile handhelds as a platform for conducting geographically distributed complex applications and in future, for deploying such applications in a scalable manner.
\end{abstract}

Categories \& Subject Descriptors: C.5.3 Microcomputers; Portable devices (e.g., laptops, personal digital assistants)

\section{General Terms: Design}

Keywords: mobile applications

Copyright is held by the author/owner(s).

MobiHeld 2010, August 30, 2010, New Delhi, India.

ACM 978-1-4503-0197-8/10/08. 


\section{Bio:}

Sanjoy Paul is Associate Vice President, General Manager-Research and Head of Convergence $\mathrm{Lab}$ at Infosys Technologies Limited where he heads research and innovation in the field of Communications, Media and Entertainment. Prior to that, he was a Research Professor at WINLAB, Rutgers University, and Founder of RelevantAd Technologies Inc. Before that Sanjoy spent five years as the Director of Wireless Networking Research at Bell Labs, Lucent Technologies, and as the CTO of two start-up companies based in New York. In a previous tenure at Bell Labs as a Distinguished Member of Technical Staff, Sanjoy was the chief architect of Lucent's IPWorX Caching and Content Distribution product line.

Sanjoy has over twenty years of technology expertise, specifically in the areas of End-to-End Protocol Design and Analysis, Mobile Wireless Networking, Quality of Service, Multicasting, Content Distribution, Media Streaming, Intelligent Caching, and Secure Commerce. He has authored a book on Multicasting, published over 100 papers in International Journals and refereed Conference Proceedings, authored over $90+$ US patents (28 granted, 60+ pending), and is the co-recipient of 1997 William R. Bennett award from IEEE Communications Society for the best original paper in IEEE/ACM Transactions on Networking.

He holds a Bachelor of Technology degree from IIT Kharagpur, India, an M.S and a Ph.D. degree from the University of Maryland, College Park, and an MBA from the Wharton Business School, University of Pennsylvania. Sanjoy is a Fellow of IEEE and a Member of the ACM. 